Schmerz 2009 $23: 109-111$

DOI 10.1007/s00482-009-0790-9

Online publiziert: 28. Februar 2009

(c) Deutsche Gesellschaft zum Studium des Schmerzes. Published by Springer

Medizin Verlag - all rights reserved 2009
E.M. Pogatzki-Zahn ${ }^{1} \cdot$ P.K. Zahnn $\cdot$ R. Sabatowski ${ }^{2}$ M. Strumpf ${ }^{3} \cdot$ S. Wirz ${ }^{4}$.

A. Wiebalck $\cdot$ H. Zirngibl ${ }^{6} \cdot$ W. Meissner ${ }^{7}$

${ }^{1}$ Klinik und Poliklinik für Anästhesiologie und operative Intensivmedizin, Universitätsklinikum Münster

${ }^{2}$ Klinik und Poliklinik für Anästhesiologie und Intensivtherapie,

Universitätsklinikum Carl Gustav Carus, Dresden

${ }^{3}$ Zentrum Anästhesiologie, Rettungs- und Intensivmedizin,

Georg-August-Universität, Göttingen

${ }^{4}$ Abteilung für Anästhesie und interdisziplinäre Intensivmedizin, Schmerztherapie,

Palliativmedizin, CURA-Krankenhaus St.-Johannes, Bad Honnef

${ }^{5}$ Klinik für Anaesthesiologie, Intensiv-, Palliativ- und Schmerzmedizin,

Berufsgenossenschaftliches Universitätsklinikum Bergmannsheil, Bochum

${ }^{6}$ Klinik für Viszeral- und Gefäßchirurgie, Helios-Klinikum Barmen, Wuppertal

${ }^{7}$ Klinik für Anästhesiologie und Intensivtherapie, Universitätsklinikum Jena

\section{Umgang mit retardierten Opioiden in der perioperativen Schmerztherapie}

\author{
Eine Stellungnahme zum neuen Warnhinweis \\ in den Fachinformationen retardierter Opioide
}

In der perioperativen Schmerztherapie konnten in den letzten 10 Jahren nicht zuletzt durch die Etablierung von Akutschmerzdiensten und einer breiteren Anwendung regionaler und patientenkontrollierter Analgesieverfahren deutliche Fortschritte erzielt werden. Trotzdem stellen aktuelle nationale und internationale Umfragen immer wieder fest, dass mehr als 50\% der Patienten über moderate bis schwere postoperative Schmerzen klagen $[1,4]$. Zu den Patientengruppen, die immer wieder durch unzureichend therapierte Schmerzen auffallen, zählen Patienten, die ein regionales oder patientenkontrolliertes Verfahren aus klinischen oder ökonomischen Gründen nicht erhalten können. Häufig handelt es sich bei den durchgeführten Operationen um kleine bis mittelgroße Eingriffe, bei denen der Aufwand und auch die potenziellen Risiken einer Regionalanästhesie nicht gerechtfertigt erscheinen. Auch Patienten, die sich einem ambulanten Eingriff unterziehen, sind häufig betroffen. Aus medizinischer und auch ökonomischer Sicht ist allerdings eine unzureichende Schmerztherapie mit weit reichenden Folgen behaftet; so stellen z. B. postoperative Schmerzen einen der Hauptgründe für eine verzögerte postoperative Entlassung oder spätere Wiedervorstellung des Patienten dar [12]. Hieraus folgt, dass es im klinischen Alltag an einem effektiven Therapiekonzept für Patienten mit zu erwartenden mittelstarken Schmerzen nach Operationen, bei denen invasivere und aufwendige Maßnahmen nicht indiziert sind, fehlt.

Eine Vielzahl von Untersuchungen konnte in den letzten Jahren zeigen, dass die planmäßige und zeitlich begrenzte orale Gabe retardierter, starker Opioide eine wirksame Therapiemöglichkeit postoperativer Schmerzen darstellen kann $[2,3,5,6,12]$. Insbesondere der Einsatz von retardiertem Oxycodon (z. B. als Oxygesic ${ }^{\circledast}$ oder in Kombination mit Naloxon als Targin ${ }^{\circledR}$ erhältlich) hat nicht zuletzt aufgrund interessanter pharmako- kinetischer Eigenschaften im Bereich der postoperativen Schmerztherapie deutlich zugenommen. Überraschend ist nun ein seit Mitte des Jahres in fast alle Fachinformationen retardierter Opioide eingefügter Warnhinweis, dass retardierte Opioide nicht für unmittelbar postoperativ auftretende Schmerzen empfohlen werden. Obgleich der genaue Wortlaut in den einzelnen Fachinfos leicht variiert, sind der Inhalt und damit die „Empfehlungen“" sehr ähnlich. Für Targin ${ }^{\circledast}$ heißt es beispielhaft:

“...Die Gabe von Targin ${ }^{\oplus}$ Retardtabletten wird präoperativ und während der ersten 12-24 Stunden postoperativ nicht empfohlen. In Abhängigkeit von Art und Umfang des chirurgischen Eingriffs, dem gewählten Anästhesieverfahren, der sonstigen Begleitmedikation sowie vom individuellen Zustand des Patienten ist der Zeitpunkt des postoperativen Einsatzes von Targin ${ }^{\oplus}$ Retardtabletten nach sorgfältiger Abwägung von Nutzen und Risiko im Einzelfall festzulegen.....“ 
Hintergrund dieses Warnhinweises soll u. a. der so genannte „Dose-dumping-Effekt " in Zusammenhang mit einer perioperativen Magenentleerungsstörung sein. Eine vorübergehende perioperative Magenentleerungsstörung könnte zu einer Überdosierung führen, wenn ein retardiertes Präparat seinen Wirkstoff bereits im Magen freisetzt, dieser jedoch erst nach Wiedereinsetzen der anterograden Magenentleerung nach distal transportiert und dann in großer Menge resorbiert wird.

Auch für nichtretardierte Opioide findet sich ein neuer Zusatz in der Fachinformation:

„Oxygesic ${ }^{\circledR}$ akut ist präoperativ und während der ersten 12-24 Stunden postoperativ nur mit Vorsicht anzuwenden. In Abhängigkeit von Art und Umfang des chirurgischen Eingriffs, dem gewählten Anästhesieverfahren, der sonstigen Begleitmedikation sowie vom individuellen Zustand des Patienten ist der Zeitpunkt des postoperativen Einsatzes von Oxyge$\mathrm{sic}^{\oplus}$ akut nach sorgfältiger Abwägung von Nutzen und Risiko im Einzelfall festzulegen."

In der Literatur sind bisher keine Fallberichte über derartige perioperative Überdosierungen durch Oxycodon (retardiert oder nichtretardiert) bekannt. Unsere eigenen Erfahrungen sowie Erfahrungen vieler Kollegen mit Schmerzkonzepten, die auf retardierten Opioiden in der (frühen) perioperativen Phase basieren, sind ausgesprochen positiv [10, 11], sodass sich beispielsweise an der Universitätsklinik Münster ein Therapiealgorithmus mit einem retardierten Opioid für Patienten nach bestimmten Operationen mit mittelschweren Schmerzen erfolgreich durchgesetzt hat (PogatzkiZahn et al., submitted). Hierbei dürfen allerdings retardierte Opioide perioperativ nicht nach Bedarf, sondern nur anhand standardisierter Analgesiekonzepte verabreicht werden. Ein solches Konzept stellt $z$. B. die planmäßige und zeitlich begrenzte Gabe von Oxycodon retard als Basisanalgetikum morgens und abends und die zusätzliche Gabe eines schnell wirksamen Opioids als Bedarfsanalgetikum (Rescue-Analgesie) bei Schmerzspitzen dar $[10,11]$. Wie bei jeder Gabe von Opioiden sind Schulungen des Stationsperso- nals, Risikoassessment und adäquates $\mathrm{Pa}$ tientenmonitoring selbstverständliche Vorraussetzungen für die Durchführung derartiger Konzepte.

Alternativen zu derartigen Konzepten mit retardierten Opioiden sind z. B. eine nicht weniger risikobehaftete intravenöse bedarfsorientierte Therapie z. B. mit Piritramid i.v. als Kurzinfusion oder die den retardierten Opioiden unterlegene Applikation oraler nichtretardierter Opioide [15]. Erste multizentrische Zahlen aus dem QUIPS-Projekt auf der Basis von mehr als 1700 Fällen demonstrieren nicht nur die weite Verbreitung der perioperativen Applikation retardierter Opioide im klinischen Alltag, sondern auch die bessere Wirksamkeit im Vergleich zur konventionellen systemischen Schmerztherapie bei gleichzeitig tendenziell eher geringeren Nebenwirkungen [8]. So betrug die maximale Schmerzintensität auf der numerischen Ratingskala (NRS) für Oxycodon vs. Piritramid und Tramadol: 4,7 bzw. 5,8 und 5,9 (11-stufige NRS), Bewegungsbeeinträchtigung und Zufriedenheit unterschieden sich ebenfalls deutlich zu Gunsten von retardiertem Oxycodon. Die Inzidenz von Müdigkeit innerhalb der ersten $24 \mathrm{~h}$ war bei der Gabe von Oxycodon retard gegenüber Piritramid oder Tramadol nicht erhöht, wie dies bei einer Überdosierung durch übermäßige Resorption zu erwarten wäre.

Es stellt sich also nun die Frage, wie mit dieser modifizierten Fachinformation umgegangen werden soll. Zunächst ist der Warnhinweis in der Produktinformation kein Verbot der Substanzanwendung oder eine "Black-box-Warnung“ aufgrund schwerwiegender Komplikationen. Es ist daher jeder ärztliche Mitarbeiter legitimiert, sich im Rahmen einer individuellen Risiko-Nutzen Abwägung („individuelle Heilbehandlung“) für die Anwendung mit retardiertem Oxycodon im Rahmen der unmittelbaren postoperativen Schmerztherapie zu entscheiden. Hierbei muss $u$. a. auch berücksichtigt werden, dass die (alternativen) konventionellen systemischen Verfahren (z. B. die Gabe intravenöser Opioide) potenziell lebensgefährliche Komplikationen nach sich ziehen können, von denen zahlreiche in der Literatur dokumentiert sind (u. a. in $[7,9,13,14])$.
Nach unserer Ansicht könnte die gegenwärtige Situation Anlass sein, eine standardisierte Aufklärung über systemische postoperative Schmerztherapieverfahren generell einzuführen, sofern dies nicht bereits geschehen ist. Deutsche und internationale Leitlinien messen einer solchen Aufklärung höchste Bedeutung zu (Empfehlungsgrad A, S3-Leitlinien 2007, „Behandlung akuter perioperativer und posttraumatischer Schmerzen“, AWMF Nr. 041/oo1), Zitat:

“...Zusammenfassend sollte die präoperative Aufklärung, Informationsvermittlung und Handlungsanleitung folgende Punkte umfassen: (...) 3. Aufklärung über Art (ggf. Anleitung zur PCA), Wirkung und Nebenwirkung der verabreichten medikamentösen Schmerztherapie unter Berücksichtigung bestehender Vorurteile gegenüber starken Schmerzmedikamenten...").

Darüber hinaus wird von einigen Experten die Ansicht geäußert, dass auch aus medikolegalen Gründen die Aufklärung über die beabsichtigte Gabe von Nichtopioiden und Opioiden aufklärungspflichtig ist, sobald substanzspezifische Risiken möglich sind (wie dies bei nahezu allen verwendeten Substanzen einschließlich der Nichtopioidanalgetika der Fall ist). Die Empfehlung zur Aufklärung über systemische Schmerztherapie betrifft deshalb nicht nur Therapiekonzepte mit retardierten Opioiden, sondern sollte unabhängig von der Wahl des Analgesieverfahrens erfolgen.

Im Vordergrund der Aufklärung sollten realistische Informationen über den zu erwartenden Schmerzverlauf sowie die Bedeutung und Möglichkeiten der Schmerzlinderung stehen. Daneben sollte der Arzt die substanzspezifischen Risiken der gewählten Verfahren (ggf. einschließlich des Warnhinweises) erwähnen und seine Entscheidung über den Einsatz erläutern. Der Patient hat danach die Möglichkeit, selbst über eine Anwendung dieser Substanzen in der perioperativen Phase zu entscheiden. Um Missverständnissen vorzubeugen, sollte vermieden werden, dass der Patient diese Informationen erst selbst aus dem Beipackzettel erfährt und sich so durch die Anwendung von Oxycodon gefährdet fühlen könnte. Nicht zur Risikoaufklärung, wohl aber zu 
den ärztlichen Beratungspflichten sollte es außerdem gehören, den Patienten auf mögliche Nebenwirkungen oder Symptome hinzuweisen und ihm mitzuteilen, wie er sich im entsprechenden Fall verhalten sollte.

\section{Fazit}

Aus unserer Sicht kann im Rahmen eines individuellen Entscheidungsprozesses durch den behandelnden Arzt ein retardiertes Opioid (z. B. retardiertes Oxycodon ohne oder mit Naloxon) und ein nichtretardiertes Opioid als Rescue-Medikation weiterhin für die unmittelbare postoperative Schmerztherapie eingesetzt werden. Eine Patientenaufklärung über Vor- und Nachteile der postoperativen systemischen Schmerztherapie, z. B. im Rahmen des Prämedikationsgesprächs, wird hierbei empfohlen.

\section{Korrespondenzadresse}

\section{Prof. Dr. E.M. Pogatzki-Zahn}

Klinik und Poliklinik für Anästhesiologie und operative Intensivmedizin, Universitätsklinikum Münster Albert-Schweitzer-Str. 33, 48129 Münster pogatzki@anit.uni-muenster.de

Interessenkonflikt. Die korrespondierende Autorin weist auf folgende Beziehungen hin: Beratungs- und Referententätigkeiten für Pfizer, Janssen-Cilag, Mundipharma, Köhler-Chemie, Wyeth, MSD, BristolMyers-Squibb.

\section{Literatur}

1. Apfelbaum JL, Chen C, Mehta SS, Gan TJ (2003) Postoperative pain experience: results from a national survey suggest postoperative pain continues to be undermanaged. Anesth Analg 97:534-540

2. Blumenthal S, Min K, Marquardt M, Borgeat A (2007) Postoperative intravenous morphine consumption, pain scores and side effects with perioperative oral controlled-release oxycodone after lumbar discectomy. Anesth Analg 105:233-237

3. De Beer JV, Winemaker MJ, Donnelly GA et al (2005) Efficacy and safety of controlled-release oxycodone and standard therapies for postoperative pain after knee or hip replacement. Can J Surg 48:277-283

4. Fletcher D, Fermanian C, Mardaye A, Aegerter P (2008) A patient-based national survey on postoperative pain management in France reveals significant achievements and persistent challenges. Pain 137:441-451

5. Ho HS (2008) Patient-controlled analgesia versus oral controlled-release oxycodone - are they interchangeable for acute postoperative pain after laparoscopic colorectal surgeries? Internat Soc Cell 74 [suppl 1]:61-65
6. Kogan A, Medalion B, Raanani E et al (2007) Early oral analgesia after fast-track cardiac anesthesia. Can J Anaesth 54:254-261

7. Lötsch J, Dudziak R, Freynhagen R et al (2006) Fatal respiratory depression after multiple intravenous morphine injections. Clin Pharmacokinet 45:10511060

8. Mescha S, Rothaug J, Meißner W (2008) Perioperative Gabe von Oxycodon: Anwendungshäufigkeit und Outcome. Schmerz 22 [suppl 2]:178

9. Musshoff F, Padosch SA, Madea B (2005) Death during patient-controlled analgesia: piritramide overdose and tissue distribution of the drug. Forensic Sci Int 154:247-251

10. Pogatzki-Zahn EM, Zahn PK (2007) Systemic analgesia: an update. Anaesthesiol Intensivmed Notfallmed Schmerzther 42:22-31

11. Pogatzki-Zahn EM, Zahn PK (2008) New substances and applications for postoperative pain therapy. Schmerz 22:353-369

12. Reuben SS, Steinberg RB, Maciolek H, Joshi W (2002) Preoperative administration of controlledrelease oxycodone for the management of pain after ambulatory laparoscopic tubal ligation surgery. J Clin Anesth 14:223-227

13. Shapiro A, Zohar E, Zaslansky R et al (2005) The frequency and timing of respiratory depression in 1524 postoperative patients treated with systemic or neuraxial morphine. J Clin Anesth 17:537-542

14. Syed S, Paul JE, Hueftlein M et al (2006) Morphine overdose from error propagation on an acute pain service. Can J Anaesth 53:586-590

15. Wirz S, Wartenberg HC, Wittmann M, Nadstawek $\mathrm{J}$ (2005) Post-operative pain therapy with oxycodone or tramadol following orthopedic surgery. A prospective, randomized, double-blind investigation. Pain Clin 17:367-376
Förderpreis für Schmerzforschung 2009 ausgeschrieben Bewerbungsfrist: 31. Mai 2009

Der von der Deutsche Gesellschaft zum Studium des Schmerzes e.V. (DGSS) und Grünenthal $\mathrm{GmbH}$ jährlich vergebene Preis fördert Ärzte, Psychologen und Naturwissenschaftler, deren Arbeiten im Bereich der anwendungsbezogenen Forschung und umgesetzten Grundlagenforschung einen wesentlichen Beitrag zur interdisziplinären praxisbezogenen Therapie akuter und chronischer Schmerzen geleistet haben. Der Preis richtet sich vorzugsweise an den wissenschaftlichen Nachwuchs. In den Kategorien „Klinische Forschung" und "Grundlagenforschung" werden jeweils ein erster und ein zweiter Preis vergeben. Die ersten Preise sind mit je 7.000 Euro, die zweiten Preise mit je 3.500 Euro ausgestattet. Die Verleihung der Preise und öffentliche Bekanntgabe der Gewinner findet anlässlich des Deutschen Schmerzkongresses 2009 in Berlin statt. Die Preisträger werden schriftlich informiert. Der Ausschreibungstext findet sich im Internet auf der Homepage der DGSS unter "Förderpreise".

Quelle:

Deutsche Gesellschaft zum Studium des Schmerzes e.V. (DGSS) www.dgss.org 